\title{
Matched Charitable Contributions: Comparative Statics and Equilibrium
}

\author{
Daniel K. Biederman \\ Department of Economics, University of North Dakota, Grand Forks, USA \\ Email: daniel.biederman@business.und.edu
}

Received August 8, 2013; revised September 6, 2013; accepted September 13, 2013

Copyright (c) 2013 Daniel K. Biederman. This is an open access article distributed under the Creative Commons Attribution License, which permits unrestricted use, distribution, and reproduction in any medium, provided the original work is properly cited.

\begin{abstract}
A small optimizing agent maximizes his utility by allocating his income to private consumption and to a contribution to a particular charity. The agent's contribution may be matched, at a particular rate, by a large agent. We provide a comprehensive comparative-statics analysis of the agent's problem, allowing for changes in the agent's income, the agent's conjecture about other agents' contributions, and the match rate. A Nash equilibrium among $n$ such agents is shown to exist if private consumption is a normal good for all agents. The equilibrium is unique if private consumption and charitable giving are normal goods for all agents.
\end{abstract}

Keywords: Charitable Contributions; Match Rate; Monotonic Transformation; Nash Equilibrium

\section{Introduction}

Fundraising efforts by charities sometimes feature an offer to match individual contributions at a particular rate, commonly called the match rate. Solicitations may inform potential contributors that a certain benefactor has offered to contribute, say, 50 cents or one dollar or two dollars for every dollar contributed by others. To the extent, match offers and other fundraising techniques are successful in generating higher levels of contributions than otherwise would be observed, they enhance the supply of certain privately-provided public goods, thereby mitigating the undersupply of such goods. However, only in recent years, economists began to devote considerable efforts to the study of fundraising methods. Karlan and List [1, p. 1774] note that fundraisers are "typically long on rules of thumb and short on hard scientific evidence, divided as to the most efficient means to attract ... dollars."

We are concerned, in this paper, with matched contributions. ${ }^{1}$ We formulate a model in which each of $n$ "small," purely-altruistic, optimizing agents allocates his income to private consumption and a contribution to a particular charitable organization. The agent is aware that his contribution, as well as the contributions of others,

${ }^{1}$ Previous theoretical and empirical studies of matched contributions include Andreoni [2], Eckel and Grossman [3], Gong and Grundy [4], Huck and Rasul [5], Karlan, List, and Shafir [6], Meier [7], and Rondeau and List [8]. will be matched at a particular rate. They formulate a Nash conjecture about the total charitable contribution of the other $n-1$ small agents. Given values of his income, his Nash conjecture, and the match rate, the agent maximizes his utility subject to his budget constraint.

The purpose of the paper is twofold. First, we aim to fill a gap in the existing literature by providing a comprehensive comparative-statics analysis of the problem faced by an agent whose charitable contribution is matched. We derive relatively simple, elasticity-based expressions for the partial derivatives of the agent's charitable contribution with respect to a change in his income, a change in his conjecture about other agents' contributions, and a change in the match rate. The signs of these partial derivatives are directly related to the elasticities of the agent's marginal rate of substitution with respect to changes in the agent's private consumption and in the total contribution to the charity.

Second, we apply our comparative-statics results to establish conditions that ensure existence and uniqueness of Nash equilibrium among the $n$ agents. These conditions overlap, in some measure, the conditions obtained by Bergstrom, Blume, and Varian [9] for the existence and uniqueness of Nash equilibrium for a class of models in which a public good is privately-provided. Our results generalize the Bergstrom et al. results in the sense that, if we set the net match rate equal to zero in our model, we obtain, essentially, the Bergstrom et al. model. Further- 
more, Bergstrom et al. offer a single set of conditions from which existence and uniqueness follow, whereas we distinguish between conditions that ensure existence and conditions that ensure existence and uniqueness.

We present the basic model and our results on individual contributions in Section 2. Nash equilibrium is the subject of Section 3. An example follows in Section 4, and we conclude with Section 5.

\section{The Model and Comparative Statics}

There are $n$ "small" agents, with small agent $i$ allocating his income, $y_{i}$, to expenditure on a composite private good, $x_{i}$, and a contribution, $c_{i}$, to a particular charitable organization. ${ }^{2}$ The organization produces a public good from the private good via a linear technology. Normalizing the price of the private good at unity, agent $i$ 's budget constraint is simply

$$
y_{i}=x_{i}+c_{i} .
$$

Apart from the $n$ small agents, there exists a benefactor (or "large" agent) who offers to match the $n$ small agents' contributions such that the total contribution to the charity, $C$, including the match, amounts to

$$
C=K \cdot \sum_{i=1}^{n} c_{i} \text {. }
$$

In (2), $K \geq 1$ is the gross match rate. In the absence of a match offer, $K=1$ and we have the ordinary case of a privately-provided public good. If $K=2$, there is a one-to-one match; if $K=3$, there is a two-to-one match; etc. The match rate is not restricted to integer-values.

We will not take up, in this paper, the problem faced by the large agent, that is, the benefactor who matches others' contributions. Instead, we regard the match rate, $K$, as exogenous. We assume small agents are motivated to contribute to the charity by "pure altruism" in the sense of Andreoni [11]. That is, agent $i$ experiences no "warm glow" from contributing; rather, his utility depends only upon his private consumption, $x_{i}$, and upon the total contribution, $C$. Agent $i$ 's utility function, $u_{i}\left(x_{i}, C\right)$, is twice continuously differentiable and strictly quasiconcave, with positive marginal utilities. We confine the analysis to the case of interior solutions $\left(x_{i}>0, c_{i}>0\right)$.

Agent $i$ formulates a fixed, Nash-type conjecture about the total contributions of the other $n-1$ small agents. ${ }^{3}$ Denoting agent $i$ 's conjecture about the contributions of others by $C_{-i} \equiv \sum_{j \neq i} c_{j}$, it follows that his ex ante conjecture about the total contribution, $C_{i}$, is

\footnotetext{
${ }^{2}$ Some models allow for multiple (rival) charitable organizations; see, for example, Apinunmahakul and Barham [10].

${ }^{3}$ The Nash--conjecture assumption is standard in the analysis of privately--provided public goods in general, and in the analysis of charitable contributions in particular; see, for example, Bergstrom, Blume, and Varian [9], Andreoni [2,11,12], and Gong and Grundy [4].
}

$$
C_{i}=K \cdot\left(c_{i}+C_{-i}\right) .
$$

Accordingly, agent $i$ 's problem is to choose $x_{i}$ and $c_{i}$ to

$$
\operatorname{Max} u_{i}\left(x_{i}, K \cdot\left(c_{i}+C_{-i}\right)\right),
$$

subject to (1). Employing the notation

$$
u_{x} \equiv \partial u_{i}\left(x_{i}, C_{i}\right) / \partial x_{i} \text { and } u_{C} \equiv \partial u_{i}\left(x_{i}, C_{i}\right) / \partial C_{i},
$$

the first-order conditions imply

$$
u_{x}\left(x_{i}, C_{i}\right)=K \cdot u_{C}\left(x_{i}, C_{i}\right) .
$$

At his optimum, the agent's marginal rate of substitution $\left(\mathrm{MRS} \equiv u_{x} / u_{C}\right)$ is equal to the gross match rate, and his optimal choices of $x_{i}$ and $c_{i}$ satisfy (1) and (4).

\subsection{Comparative Statics}

We are interested in how the agent responds to changes in $y_{i}, C_{-i}$, and $K$. The total differentials of (1) and (3) are

$$
\mathrm{d} y_{i}=\mathrm{d} x_{i}+\mathrm{d} c_{i}
$$

and

$$
\mathrm{d} C_{i}=\left(\mathrm{d} c_{i}+\mathrm{d} C_{-i}\right) K+\left(C_{i} / K\right) \mathrm{d} K,
$$

respectively. Employing the notation

$$
u_{x x} \equiv \partial^{2} u_{i}\left(x_{i}, C_{i}\right) / \partial x_{i}^{2}, u_{x C} \equiv \partial^{2} u_{i}\left(x_{i}, C_{i}\right) / \partial x_{i} \partial C_{i},
$$

etc., the total differential of (4) is

$$
u_{x x} \mathrm{~d} x_{i}+u_{x C} \mathrm{~d} C_{i}=K \cdot\left(u_{C X} \mathrm{~d} x_{i}+u_{C C} \mathrm{~d} C_{i}\right)+u_{C} \mathrm{~d} K .
$$

We wish to rewrite (7) in elasticity form. First, we will identify four elasticities of marginal utility. Subsequently, we will relate those four elasticities to two elasticities of the MRS. We use $\eta_{x x}$ and $\eta_{x C}$ to denote the elasticities of $u_{x}$ with respect to $x_{i}$ and $C_{i}$, respectively:

$$
\begin{aligned}
& \eta_{x x} \equiv \frac{\partial u_{x}}{\partial x_{i}} \cdot \frac{x_{i}}{u_{x}}=\frac{x_{i} u_{x x}}{u_{x}} \\
& \text { and } \\
& \eta_{x C} \equiv \frac{\partial u_{x}}{\partial C_{i}} \cdot \frac{C_{i}}{u_{x}}=\frac{C_{i} u_{x C}}{u_{x}} .
\end{aligned}
$$

We use $\eta_{C_{X}}$ and $\eta_{C C}$ to denote the elasticities of $u_{C}$ with respect to $x_{i}$ and $C_{i}$, respectively:

$$
\begin{aligned}
& \eta_{C X} \equiv \frac{\partial u_{C}}{\partial x_{i}} \cdot \frac{x_{i}}{u_{C}}=\frac{x_{i} u_{C X}}{u_{C}} \\
& \text { and } \\
& \eta_{C C} \equiv \frac{\partial u_{C}}{\partial C_{i}} \cdot \frac{C_{i}}{u_{C}}=\frac{C_{i} u_{C C}}{u_{C}} .
\end{aligned}
$$

The elasticity of the agent's MRS with respect to $x_{i}$, 
which we denote by $\varepsilon_{x}$, is equal to $\eta_{x x}-\eta_{C x}$ :

$$
\begin{aligned}
\varepsilon_{x} & \equiv \frac{\partial\left(u_{x} / u_{C}\right)}{\partial x_{i}} \cdot \frac{x_{i}}{u_{x} / u_{C}} \\
& =\frac{u_{C} u_{x x}-u_{x} u_{C x}}{u_{C}^{2}} \cdot \frac{x_{i} u_{C}}{u_{x}}=\eta_{x x}-\eta_{C x} .
\end{aligned}
$$

Similarly, the elasticity of the agent's MRS with respect to $C_{i}$, which we denote by $\varepsilon_{C}$, is equal to $\eta_{x C}-\eta_{C C}:$

$$
\begin{aligned}
\varepsilon_{C} & \equiv \frac{\partial\left(u_{x} / u_{C}\right)}{\partial C_{i}} \cdot \frac{C_{i}}{u_{x} / u_{C}} \\
& =\frac{u_{C} u_{x C}-u_{x} u_{C C}}{u_{C}^{2}} \cdot \frac{C_{i} u_{C}}{u_{x}}=\eta_{x C}-\eta_{C C} .
\end{aligned}
$$

Using (4) and (8)-(11), we can rewrite (7) as follows:

$$
\varepsilon_{x} \cdot \frac{\mathrm{d} x_{i}}{x_{i}}+\varepsilon_{C} \cdot \frac{\mathrm{d} C_{i}}{C_{i}}=\frac{\mathrm{d} K}{K} .
$$

We use (5), (6), and (12) to obtain

$$
\begin{aligned}
\delta \mathrm{d}_{i}= & -\varepsilon_{x} C_{i} \mathrm{~d} y_{i}-\varepsilon_{C} K x_{i} \mathrm{~d} C_{-i} \\
& +\left(1-\varepsilon_{C}\right)\left(C_{i} x_{i} / K\right) \mathrm{d} K,
\end{aligned}
$$

where

$$
\delta \equiv \varepsilon_{C} K x_{i}-\varepsilon_{x} C_{i} .
$$

Using (13), we easily obtain the partial derivatives that are central to our comparative-statics analysis:

$$
\begin{aligned}
& \partial c_{i} / \partial y_{i}=-\left(C_{i} / \delta\right) \varepsilon_{x}, \\
& \partial c_{i} / \partial C_{-i}=-\left(K x_{i} / \delta\right) \varepsilon_{C}, \\
& \partial c_{i} / \partial K=\left(C_{i} x_{i} / K \delta\right)\left(1-\varepsilon_{C}\right) .
\end{aligned}
$$

The second-order sufficient condition for a maximum is $\delta>0$, and that condition is ensured by the assumption of strictly-quasiconcave utility. ${ }^{4}$

In view of (15), $\partial c_{i} / \partial y_{i}>0$ if and only if ("iff") $\varepsilon_{x}<0$. This is the case when the agent's MRS, at the optimum, decreases with his private consumption. Furthermore, using (14) and (16), we can determine that $\varepsilon_{x}<0$ iff $\partial c_{i} / \partial C_{-i}>-1$. Thus, to say that an agent's charitable contribution, $c_{i}$, is normal is to say that when that agent conjectures an increase in the contributions of other agents, his best reply is such that his conjecture about the total contribution, $C_{i}$, increases.

Noting that $\partial x_{i} / \partial y_{i}=1-\partial c_{i} / \partial y_{i}$, it follows from (14) and (15) that $\partial x_{i} / \partial y_{i}>0$ iff $\varepsilon_{C}>0$. This is the case when the agent's MRS, at the optimum, increases with his conjecture about the total contribution. Using (16), it

${ }^{4}$ Ordinarily, the quasiconcavity condition for the problem at hand would be written as $-u_{x x}+2 K u_{x C}-K^{2} u_{C C}>0$. It can be shown that $\delta=\left(C_{i} x_{i} / u_{x}\right)\left(-u_{x x}+2 K u_{x C}-K^{2} u_{C C}\right)$. follows as well that $\partial c_{i} / \partial C_{-i}<0$ iff $\varepsilon_{C}>0$. To say that an agent's private consumption is normal, then, is to say that an increase in his conjecture about others' contributions prompts him to decrease his own contribution.

From (17) it is clear that the agent will increase his contribution in response to an increase in the match rate iff his MRS is inelastic with respect to his conjecture about the total contribution (that is, iff $\varepsilon_{C}<1$ ). We showed directly above that $\varepsilon_{C}>0$ is necessary and sufficient for normality of private consumption and for a decreasing best reply. However, we have no a priori knowledge about the sign of $1-\varepsilon_{C}$. Indeed, it seems entirely reasonable to believe that, depending on an agent's income and preferences, $c_{i}$ may increase, decrease, or remain unchanged in response to an increase in the match rate. ${ }^{5}$

We can summarize our comparative-statics results as follows. For agent $i$, we have

$$
0<\partial x_{i} / \partial y_{i}<1,0<\partial c_{i} / \partial y_{i}<1 \text {, and }-1<\partial c_{i} / \partial C_{-i}<0
$$

iff $\varepsilon_{x}<0$ and $\varepsilon_{C}>0$. It is worth noting that these last two inequalities also ensure satisfaction of the secondorder sufficient condition for the agent's problem.

\subsection{ALEP Complementarity}

For a particular agent, private consumption and the total charitable contribution are said to be Auspitz-LiebenEdgeworth-Pareto (ALEP) complements if $u_{x C}>0$, and weak ALEP complements if $u_{x C} \geq 0$. It has been widely-acknowledged that the primary limitation of this notion of complementarity, which originated with Auspitz and Lieben [13], is that, in general, the signs of the second-order partial derivatives of a utility function are not invariant with respect to monotonic transformations. Nevertheless, in many problems the assumption of ALEP complementarity (or weak ALEP complementarity) allows for stronger comparative-statics results than are obtainable without the assumption; see, for example, Chipman [14] and Weber [15].

In our model, if we assume that marginal utilities are diminishing $\left(u_{x x}<0\right.$ and $\left.u_{C C}<0\right)$ and that $u_{x C} \geq 0$, then $\varepsilon_{x}<0$ and $\varepsilon_{C}>0$ and, consequently, all of the desirable" comparative-statics results-normality of $x_{i}$ and of $C_{i}$, etc.-are ensured. However, the assumption that $u_{x C} \geq 0$ is too strong. One can find strictly-quasiconcave utility functions $u(x, C)$ for which $u_{x C}<0$ and for which $\varepsilon_{x}<0$ and $\varepsilon_{C}>0$.

Moreover, the elasticities $\varepsilon_{x}$ and $\varepsilon_{C}$ are invariant to monotonic transformations. Suppose we start with a utility function $u(x, C)$ for which $u_{x x}<0, u_{C C}<0$, and $u_{x C} \geq 0$, so that $\varepsilon_{x}<0$ and $\varepsilon_{C}>0$. A monotonic

${ }^{5}$ Without probing the matter in detail, Gong and Grundy [4] note that individual contributions can either increase or decrease with the match rate. We provide an illustrative example in Section 4 below. 
transformation $\tilde{u}(x, C)=\phi(u(x, C)) \quad\left(\right.$ where $\phi^{\prime}>0$ ) may or may not preserve the inequality $u_{x C} \geq 0$, but it is certain to preserve the values of the MRS elasticities. In turn, it follows that the transformation preserves the agent's demand functions.

\section{Nash Equilibrium}

In this section we apply the comparative-statics results derived in Section 2 to the existence and uniqueness of Nash equilibrium among the $n$ agents.

Proposition 1. Suppose $c_{i}$ is continuous in $C_{-i}$ for all $i=1,2, \cdots, n$. Then, for given values of $y_{i}(i=1,2, \cdots, n)$ and $K$ :

(A) a Nash equilibrium exists if private consumption is a normal good for all agents;

(B) a unique Nash equilibrium exists if private consumption and the charitable contribution are normal goods for all agents.

Proof. We apply Theorems 2.7 and 2.8 from Vives [16]. In addition to the continuity requirement, it is required that agents' best replies depend only on the aggregate actions of other agents. This requirement is clearly met. A further requirement is that agents' strategy spaces must be compact. This requirement is met since $0 \leq c_{i} \leq y_{i}$ for all $i$. Then Vives' Theorem 2.7 asserts existence of an equilibrium if best replies are strictly decreasing, as is the case when $x_{i}$ is normal. This proves (A). Vives' Theorem 2.8 asserts existence and uniqueness of equilibrium, provided $-1<\partial c_{i} / \partial C_{-i}<0$ for all $i$, as is the case when $x_{i}$ and $c_{i}$ are normal. This proves (B). Q.E.D.

Note that Proposition 1 was established without regard to the sign or magnitude of $\partial c_{i} / \partial K$. However, we can establish existence of equilibrium for cases in which agents respond to an increase in the match rate by decreasing their individual contributions or by leaving them unchanged.

Proposition 2. Suppose $c_{i}$ is continuous in $C_{-i}$ and $\partial c_{i} / \partial K \leq 0$ for all $i=1,2, \cdots, n$. Then, for given values of $y_{i}(i=1,2, \cdots, n)$ and $K$, a Nash equilibrium exists.

Proof. It follows from (16) and (17) that if $\partial c_{i} / \partial K \leq 0$, then $\partial c_{i} / \partial C_{-i}<0$. Applying Vives' [16] Theorem 2.7, we then have existence of an equilibrium. Q.E.D.

As we noted in Section 1, our results on Nash equilibrium are related to the results obtained by Bergstrom, Blume, and Varian [9] for the case of a privately-provided good. Again, however, the Bergstrom et al. model does not focus specifically on charitable contributions, and as such there is no match rate in that model. Furthermore, Bergstrom et al. offered a single set of conditions that ensures existence and uniqueness of equilibrium, whereas we have disentangled that set of condi- tions.

\section{An Example}

Consider the constant-elasticity (CE) utility function:

$$
u\left(x_{i}, C\right)= \begin{cases}\frac{1}{1-\sigma} \cdot\left(x_{i}^{1-\sigma}+\beta C^{1-\sigma}\right) & \text { if } 0<\sigma<1 \text { or if } \sigma>1 \\ \ln x_{i}+\beta \ln C & \text { if } \sigma=1\end{cases}
$$

where $\beta>0$. Let $\gamma \equiv 1 / \sigma$. Using (1) and (4), agent $i$ 's optimal contribution is given by

$$
c_{i}=\frac{\beta^{\gamma} y_{i}-K^{1-\gamma} C_{-i}}{\beta^{\gamma}+K^{1-\gamma}} .
$$

\subsection{Comparative Statics}

It is straightforward to show, for the CE function, that $\varepsilon_{x}=-\sigma$ and $\varepsilon_{C}=\sigma$. Expressions for the partial derivatives $\partial c_{i} / \partial y_{i}, \partial c_{i} / \partial C_{-i}$, and $\partial c_{i} / \partial K$ can be obtained either through direct differentiation of (18) or by the application of (14) - (17). We present the partials in both forms:

$$
\begin{aligned}
& \partial c_{i} / \partial y_{i}=\frac{\beta^{\gamma}}{\beta^{\gamma}+K^{1-\gamma}}=\frac{C_{i}}{K x_{i}+C_{i}}, \\
& \partial c_{i} / \partial C_{-i}=-\frac{K^{1-\gamma}}{\beta^{\gamma}+K^{1-\gamma}}=-\frac{K x_{i}}{K x_{i}+C_{i}} \\
& \partial c_{i} / \partial K=\frac{\beta^{\gamma}(\gamma-1)\left(y_{i}+C_{-i}\right)}{K^{\gamma}\left(\beta^{\gamma}+K^{1-\gamma}\right)^{2}}=\frac{C_{i} x_{i}(1-\sigma)}{\sigma K\left(K x_{i}+C_{i}\right)} .
\end{aligned}
$$

It is clear that

$$
0<\partial c_{i} / \partial y_{i}<1 \text { and }-1<\partial c_{i} / \partial C_{-i}<0
$$

for all values of $\sigma$, and that $\partial c_{i} / \partial K \gtreqless 0$ as $\sigma \lesseqgtr 1$ (that is, as $\gamma \gtreqless 1$ ).

\subsection{ALEP Complementarity}

The CE utility function $u$ is additively separable, with $u_{x C}=0$. Accordingly, we refer to $x$ and $C$ as ALEP independents in this case. Now let $\tilde{u}=\phi(u)=-u^{-1}$. This increasing (and concave) transformation results in $\tilde{u}_{x C}=-2 /\left[u^{3}\left(x_{i} C_{i}\right)^{\sigma}\right]<0$, so $x$ and $C$ are now ALEP substitutes. Nevertheless, the MRS elasticities are unchanged ( $\tilde{\varepsilon}_{x}=\varepsilon_{x}=-\sigma$ and $\tilde{\varepsilon}_{C}=\varepsilon_{C}=\sigma$ ), leaving the agent's demand functions unchanged as well.

\subsection{Nash Equilibrium}

If all agents' utility functions are of the CE type, then private consumption and the charitable contribution are 
normal for all agents, and Proposition 1, Part B asserts existence and uniqueness of Nash equilibrium. Suppose, for instance, that the $n$ agents are identical in that they all have the same CE utility function and the same level of income, $y$. Setting $C_{-i}=(n-1) c_{i}$ in (18), we obtain the Nash-equilibrium contribution, $c^{*}$, for each agent:

$$
c^{*}=\frac{\beta^{\sigma} y}{\left(\beta^{\sigma}+n K^{1-\sigma}\right)} .
$$

It can be verified that the equilibrium total contribution, $C^{*}=K n c^{*}$, increases with $K$, irrespective of the value of $\sigma$.

\section{Summary and Conclusions}

We have focused on purely-altruistic, utility-maximizing agents who allocate their incomes to private consumption and to charitable contributions that are matched at an exogenous rate. We showed that the nature of an agent's response to a change in his income is inexorably linked to the nature of his best reply to a change in the contributions of other agents. We also derived a general characterization of an agent's response to a change in the match rate in terms of the elasticity of the agent's MRS with respect to a change in his conjecture about the total contribution. These comparative-statics results are useful in establishing conditions that ensure existence and uniqueness of Nash equilibrium for the case of $n$ agents.

A more general model of matched contributions might allow for an endogenous match rate. For example, the match rate might be chosen by a utility-maximizing benefactor who is cognizant of how small contributors respond to changes in that rate. In addition, agents may be driven to contribute by motives other than pure altruism. Finally, in an environment that features heterogeneous agents, some of those agents may find it optimal to refrain altogether from contributing at certain match rates. Consideration of these factors makes for ongoing research.

\section{REFERENCES}

[1] D. Karlan and J. A. List, "Does Price Matter in Charitable Giving? Evidence from a Large-Scale Natural Field Experiment," American Economic Review, Vol. 97, No. 5, 2007, pp. 1774-1793. http://dx.doi.org/10.1257/aer.97.5.1774

[2] J. Andreoni, "Leadership Giving in Charitable Fund-Raising,” Journal of Public Economic Theory, Vol. 8, No. 1, 2006, pp. 1-22. http://dx.doi.org/10.1111/j.1467-9779.2006.00250.x
[3] C. C. Eckel and P. J. Grossman, "Subsidizing Charitable Contributions: A Natural Field Experiment Comparing Matching and Rebate Subsidies," Experimental Economics, Vol. 11, No. 3, 2008, pp. 234-252. http://dx.doi.org/10.1007/s10683-008-9198-0

[4] N. Gong and B. D. Grundy, "Charitable Fund Raising: Matching Grants or Seed Money,” Unpublished Manuscript, 2011.

[5] S. Huck and I. Rasul, "Matched Fundraising: Evidence from a Natural Field Experiment," Journal of Public Economics, Vol. 95, No. 5-6, 2011, pp. 351-362. http://dx.doi.org/10.1016/j.jpubeco.2010.10.005

[6] D. Karlan, J. A. List and E. Shafir, "Small Matches and Charitable Giving: Evidence from a Natural Field Experiment,” Journal of Public Economics, Vol. 95, No. 5-6, 2011, pp. 344-350. http://dx.doi.org/10.1016/j.jpubeco.2010.11.024

[7] S. Meier, "Do Subsidies Increase Charitable Giving in the Long Run? Matching Contributions in a Field Experiment," Journal of the European Economic Association, Vol. 5, No. 6, 2007, pp. 1203-1222. http://dx.doi.org/10.1162/JEEA.2007.5.6.1203

[8] D. Rondeau and J. A. List, "Matching and Challenge Gifts to Charity: Evidence from Laboratory and Natural Field Experiments,” Experimental Economics, Vol. 11, No. 3, 2008, pp. 253-257. http://dx.doi.org/10.1007/s10683-007-9190-0

[9] T. Bergstrom, L. Blume and H. Varian, "On the Private Provision of Public Goods,” Journal of Public Economics, Vol. 29, 1986, pp. 25-49. http://dx.doi.org/10.1016/0047-2727(86)90024-1

[10] A. Apinunmahakul and V. Barham, "Strategic Interaction and Charitable Fundraising," Modern Economy, Vol. 3, 2012, pp. 338-345. http://dx.doi.org/10.4236/me.2012.33044

[11] J. Andreoni, "Giving with Impure Altruism: Applications to Charity and Ricardian Equivalence," Journal of Political Economy, Vol. 97, No. 6, 1989, pp. 1447-1458. http://dx.doi.org/10.1086/261662

[12] J. Andreoni, "Toward a Theory of Charitable FundRaising,” Journal of Political Economy, Vol. 106, No. 6, 1998, pp. 1186-1213. http://dx.doi.org/10.1086/250044

[13] R. Auspitz and R. Lieben, "Untersuchungen über die Theorie des Preises,” Duncker \& Humblot, Leipzig, 1889.

[14] J. S. Chipman, “An Empirical Implication of AuspitzLieben-Edgeworth-Pareto Complementarity,” Journal of Economic Theory, Vol. 14, No. 1, 1977, pp. 228-231. http://dx.doi.org/10.1016/0022-0531(77)90096-5

[15] C. E. Weber, "Two Further Implications of Auspitz-Lieben-Edgeworth-Pareto Complementarity,” Economics Letters, Vol. 67, No. 3, 2000, pp. 289-295. http://dx.doi.org/10.1016/S0165-1765(99)00277-3

[16] X. Vives, "Oligopoly Pricing: Old Ideas and New Tools," MIT Press, Cambridge, 1999. 